\title{
Heat Distribution in Rectangular Fins Using Efficient Finite Element and Differential Quadrature Methods
}

\author{
ShahNor BASRI, M. M. FAKIR, F. MUSTAPHA, D. L. A. MAJID, A. A. JAAFAR \\ Department of Aerospace Engineering, University Putra Malaysia, Putra, Malaysia \\ E-mail: shahnor@eng.upm.edu \\ Received September 2, 2009; revised September 24, 2009; accepted September 28, 2009
}

\begin{abstract}
Finite element method (FEM) and differential quadrature method (DQM) are among important numerical techniques used in engineering analyses. Usually elements are sub-divided uniformly in FEM (conventional FEM, CFEM) to obtain temperature distribution behavior in a fin or plate. Hence, extra computational complexity is needed to obtain a fair solution with required accuracy. In this paper, non-uniform sub-elements are considered for FEM (efficient FEM, EFEM) solution to reduce the computational complexity. Then this EFEM is applied for the solution of one-dimensional heat transfer problem in a rectangular thin fin. The obtained results are compared with CFEM and efficient DQM (EDQM), with non-uniform mesh generation). It is found that the EFEM exhibit more accurate results than CFEM and EDQM showing its potentiality.
\end{abstract}

Keywords: Efficient Finite Element Method, Efficient Differential Quadrature Method, Heat Transfer Problem

\section{Introduction}

Presently there are many numerical solution techniques known to the computational mechanics community. FEM is one of those numerical solution techniques to solve structural, mechanical, heat transfer, and fluid dynamics which arise in problems of engineering and physical sciences [1-5]. Here, conventional FEM (CFEM) means the used elements are of same size and uniformly distributed. In its application to the solution of engineering problems, the finite element discretization has been implemented almost to the spatial problems. For dynamic or time dependent problems whose solutions as functions of time are of interest, a step by step procedure of finite difference is usually employed with computational complexity.

For heat transfer problems, rapid changes of heat/temperature distributions take place near the element boundary (and at the boundary). It is very important to know these temperature change behavior of an element prior to its use. Hence, to get an actual picture using FEM, the element is usually subdivided into very small sub-elements uniformly (conventional FEM, CFEM), which leads to huge amount of complexity, memory consumption and computational time [6]. Otherwise, error flow occurs with unreliable results $[1,2,6]$.

On the other hand, to get a clear picture about the temperature changes near (and at) the element boundary, better to subdivide the elements into very small subelements at the boundary only, followed by relatively bigger elements gradually towards the mid-point of the element non-uniformly (efficient FEM, EFEM). This may serve the intended purpose without any additional burden and this is highlighted in this paper with improved accuracy (approximately 65\%) compared to CFEM. Hence, here, focus is given to develop and apply efficient (non-uniform mesh density) nodal points distribution algorithm for automatic mesh (elements) generation to optimize CFEM solution.

DQM is another numerical solution technique to solve above mentioned problems efficiently [7-13]. The essence of the DQM is that the partial derivative of a function is approximated by a weighted linear sum of the function values at given discrete points. Bellman and Casti $[7,8]$ developed this numerical solution technique in the early 1970s and since then, the technique has been successfully employed in a variety of problems in engineering and physical sciences. To make the DQM more efficient with less computational complexity, efficient DQM (EDQM) was proposed in [11-13] with non-uniformly distributed mesh points.

Hence, in this paper, one-dimensional (1-D) heat conduction problems in a thin rectangular fin are solved using EFEM by means of the accurate discretization and solver (code) and then the results are compared with 
CFEM and EDQM to verify EFEM efficiency.

The paper is organized as follows. Section II presents the governing equation with efficient FEM rules, followed by simulation set-up and assumptions, results and discussions, and finally conclusion of the paper.

\section{The One-Dimensional Efficient Finite Element Method}

Here, the considered one dimensional (1-D) heat conduction problem is $[2,3,14-18]$

$$
\frac{d}{d x}\left(k \frac{d T}{d x}\right)+Q=0
$$

with the boundary conditions $\left.T\right|_{x=0}=T_{0}$ and $\left.q\right|_{x=L}=h\left(T_{L}-T_{\infty}\right)$ as shown in Figure 1. Here, heat flux $q=-k \frac{d t}{d x}$. Figure 1 shows the 1-D element discretization in the x-direction. The temperature $T$ at various nodal points are the unknowns except at node 1, where, $T_{1}=T_{0}$ with initial temperature $T_{0}$. Within a typical element ' $e i$ or $e$ ' the local node numbers are $i$ and $i+1$ with coordinates $x_{i}$ and $x_{i+1}$ and element length, $l_{e i}=x_{i+1}-x_{i}$. For example, $e 1$ whose local node numbers are 1.and 2 with coordinates $x_{1}$ and $x_{2}$, and element length $l_{e 1}=x_{2}-x_{1}$ respectively.

An one-dimensional thin rectangular fin as shown in Figure 2 is considered here. Heat is transmitted along its length by conduction and dissipated from its lateral surfaces to the surroundings by convection. The governing equation for the temperature in the fin is given in Equation (1).

The parameter, $M$ is given by $M^{2}=\frac{h p}{k A_{C}}$, where, $\mathrm{p}$ is the fin perimeter (meter) and Ac is the cross sectional area of the fin $\left[\right.$ meter $\left.^{2}\right]$. Fin length, width and thickness are $L, w$ and $t$ respectively.

In this case, $q=h\left(T-T_{\infty}\right)=-k \frac{d T}{d x}, p=2(w+t)$, $A_{C}=w \times t$ and $\frac{p}{A_{C}}=\frac{2(w+t)}{w \times t} \approx \frac{2}{t}$. The convection heat loss in the fin is equivalent to negative heat source and can be expressed as follows:

$$
Q=-\frac{(p d x) h\left(T-T_{\infty}\right)}{A_{C} d x}=-\frac{p h}{A_{C}}\left(T-T_{\infty}\right)
$$

Now Equation (1) becomes

$$
\frac{d}{d x}\left(k \frac{d T}{d x}\right)-\frac{p h}{A_{C}}\left(T-T_{\infty}\right)=0
$$

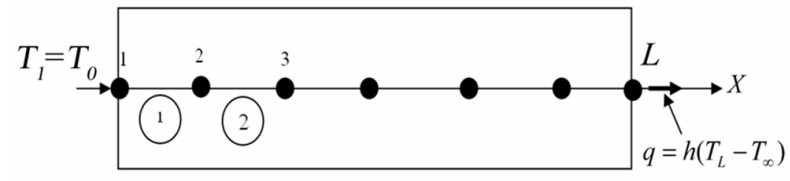

Figure 1. Boundary conditions for 1-D heat conduction.

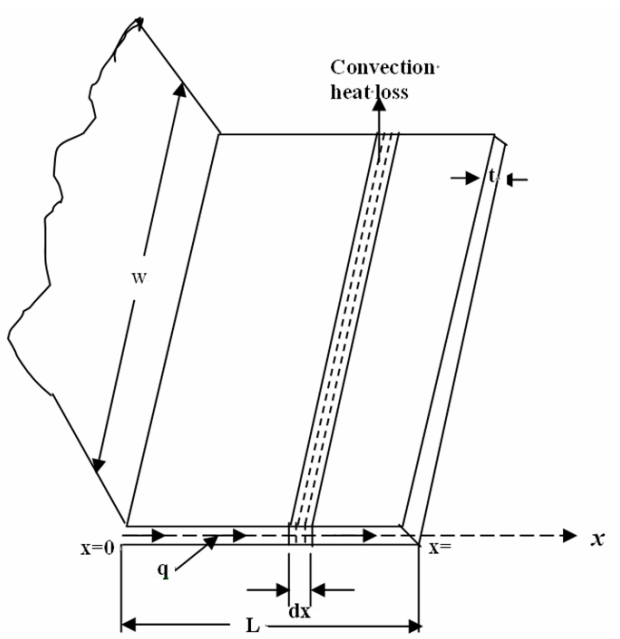

Figure 2. Thin rectangular fin.

To calculate the approximate solution $T$, the mathematical formulation using Galerkin's approach [2,3] is

$$
\int_{0}^{L} \varphi\left[\frac{d}{d x}\left(k \frac{d T}{d x}-\frac{p h}{A_{C}}\left(T-T_{\infty}\right)\right)\right] d x=0
$$

where $\varphi$ is a test function constructed from the same basis functions as those of $T$, with $\varphi(0)=0$.

Integrating by parts Equation (3) becomes,

$$
\left.\varphi k \frac{d T}{d x}\right|_{0} ^{L}-\int_{0}^{L} k \frac{d \varphi}{d x} \frac{d T}{d x} d x-\int_{0}^{L} \varphi \frac{p h}{A_{C}}\left(T-T_{\infty}\right) d x=0
$$

The $1^{\text {st }}$ term of Equation (4) is,

$$
\left.\varphi k \frac{d T}{d x}\right|_{0} ^{L}=\varphi(L) k(L) \frac{d T}{d x}(L)-\varphi(0) k(0) \frac{d T}{d x}(0)
$$

Since $\varphi(0)=0$ and $q=-k(L) \frac{d T}{d x}(L)=h\left(T_{L}-T_{\infty}\right)$, we get, $\left.\varphi k \frac{d T}{d x}\right|_{0} ^{L}=-\varphi(L) h\left(T_{L}-T_{\infty}\right)$

Equation (4) becomes

$$
-\varphi(L) h\left(T_{L}-T_{\infty}\right)-\int_{0}^{L} k \frac{d \varphi}{d x} \frac{d T}{d x} d x-\int_{0}^{L} \varphi \frac{p h}{A_{C}}\left(T-T_{\infty}\right) d x=0
$$

A global virtual temperature vector is defined as $\psi=\left[\psi_{1}, \psi_{2}, \psi_{3}, \ldots, \psi_{L}\right]^{T}$ then within each element, th 
test function becomes

$$
\varphi(i)=N_{i} \psi_{i}
$$

Here, $N$ is the element shape function and $N_{L}=1$ at the element boundary [2] (Figure 1). Therefore Equation (7) gives

$$
\begin{gathered}
\varphi(L)=[N \psi]_{L}=\psi_{L} \\
\mathbf{B}_{T}^{\mathbf{T}} \mathbf{B}_{T}=\frac{1}{x_{i+1}-x_{i}}\left[\begin{array}{r}
-1 \\
1
\end{array}\right] \times \frac{1}{x_{i+1}-x_{i}}[ \\
k_{T}=\frac{k_{e i} l_{e i}}{2} \int_{-1}^{1} \mathbf{B}_{T}^{\mathbf{T}} \mathbf{B}_{T} d \xi=\frac{k_{e i}}{l_{e i}}\left[\begin{array}{lr}
1 & -1 \\
-1 & 1
\end{array}\right]
\end{gathered}
$$

According to Reference [2], $\frac{d T}{d x}=\mathbf{B}_{T} \mathbf{T}^{e}$, we have $\frac{d \varphi}{d x}=\mathbf{B}_{T} \psi$

For matrix multiplication validity, we have $\left(\frac{d \varphi}{d x}\right)^{T} \times\left(\frac{d T}{d x}\right)=\left(\mathbf{B}_{T}^{T} \boldsymbol{\psi}^{T}\right)\left(\mathbf{B}_{\mathbf{T}} \mathbf{T}^{e i}\right)$ and $1]=\frac{1}{\left(x_{i+1}-x_{i}\right)^{2}}\left[\begin{array}{rr}1 & -1 \\ -1 & 1\end{array}\right]=\frac{1}{\left(l_{e i}\right)^{2}}\left[\begin{array}{rr}1 & -1 \\ -1 & 1\end{array}\right]$

The element conductivity matrix is

where, $\xi$ varies from -1 to +1 and $\xi=\frac{2}{x_{i+1}-x_{i}}\left(x-x_{i}\right)-1$ with $d \xi=\frac{2}{x_{i+1}-x_{i}} d x$.

$$
-\psi_{l} h\left(T_{L}-T_{\infty}\right)-\sum_{e i} \boldsymbol{\psi}^{\mathbf{T}}\left(\frac{k_{e i} l_{e i}}{2} \int_{-1}^{1} \mathbf{B}_{T}^{\mathbf{T}} \mathbf{B}_{T} d \xi\right) \mathbf{T}^{e}+\sum_{e i} \boldsymbol{\psi}^{\mathbf{T}} \frac{Q_{e i} l_{e i}}{2} \int_{-1}^{1} \mathbf{N}^{\mathbf{T}} d \xi=0
$$

or

$$
\boldsymbol{\psi}^{\mathbf{T}} \mathbf{K}_{T} \mathbf{T}+\psi_{L} h T_{L}=\boldsymbol{\psi}^{\mathbf{T}} \mathbf{R}+\psi_{L} h T_{\infty}
$$

where, global matrices $\mathbf{K}_{T}, \mathbf{R}, \boldsymbol{\psi}^{\mathbf{T}}$ and $\mathbf{T}$ are respectively,

$$
\begin{aligned}
& \mathbf{K}_{T}=\sum_{e i} \frac{k_{e i} l_{e i}}{2} \int_{-1}^{1} \mathbf{B}_{T}^{\mathrm{T}} \mathbf{B}_{T} d \xi=\left[\begin{array}{cccccc}
K_{11} & K_{12} & K_{13} & \ldots & K_{1 L} & \\
K_{21} & K_{22} & K_{23} & \ldots & K_{2 L} & \\
K_{31} & K_{32} & K_{33} & \ldots & K_{3 L} & \\
\cdots \cdots \cdots \cdots \cdots \cdots \cdots \cdots \cdots \cdots \cdots \cdots \cdots \cdots \cdots & \cdots \cdots \cdots \cdots \cdots \\
K_{L 1} & K_{L 2} & K_{L 2} & \cdots & & K_{L L}
\end{array}\right] \\
& \mathbf{R}=\sum_{e i} \frac{Q_{e i} l_{e i}^{1}}{2} \int_{-1}^{1} \mathbf{N}^{\mathbf{T}} d \xi=\left[\begin{array}{lllll}
R_{1} & R_{2} & R_{3} & \ldots & R_{L}
\end{array}\right]^{\mathbf{T}}
\end{aligned}
$$

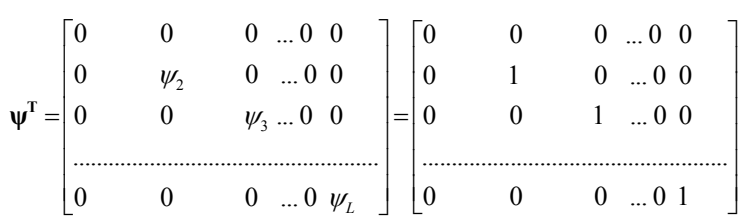

and $\mathbf{T}=\left(\begin{array}{lllll}T_{1} & T_{2} & T_{3} & \ldots & T_{L}\end{array}\right)^{\mathbf{T}}$ with $T_{1}=T_{0}=$ constant.

Finally, the global matrix is formed and the Equation (12) becomes
The element heat rate vector due to the heat source is

$$
\mathbf{R}=\mathbf{r}_{Q}=\frac{Q_{e i} l_{e i}}{2} \int_{-1}^{1} \mathbf{N}^{\mathbf{T}} d \xi=\frac{Q_{e i} l_{e i}}{2}\left[\begin{array}{l}
1 \\
1
\end{array}\right]
$$

Now, Equation (2) can be transformed into 


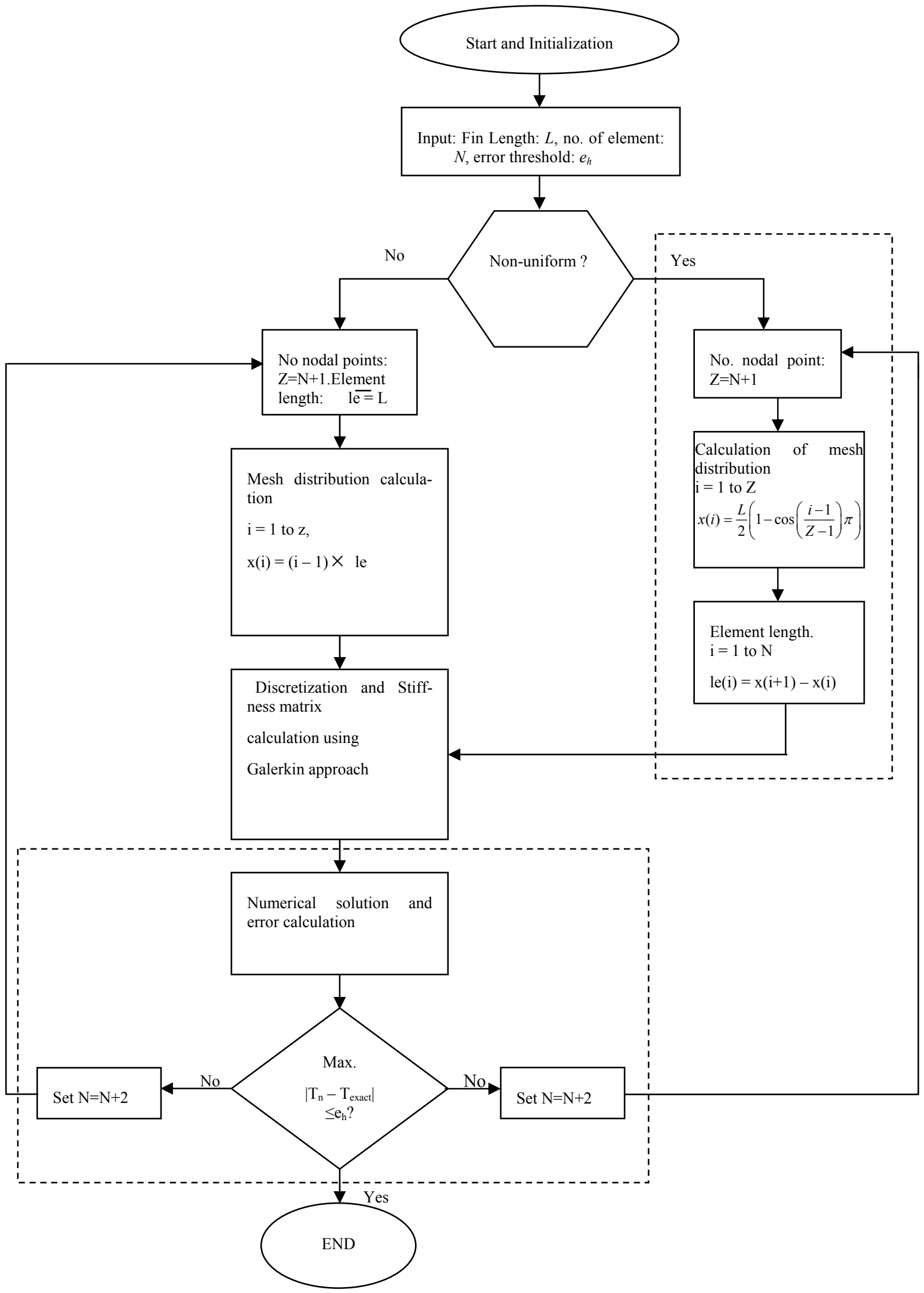

Figure 3. Efficient discretization and solution rule for 1-D FEM 


$$
T=T_{0} \quad \text { at } x=0
$$

$q=0$ at $x=L$, where $L$ is the length of the fin.

In this case, the final form of the global matrix in Equation (16) becomes

$$
\left(\begin{array}{cccc}
A_{22} & A_{23} & \ldots & A_{2 L} \\
A_{32} & A_{33} & \ldots & A_{3 L} \\
\ldots & \ldots & \ldots & \ldots \\
A_{L 2} & A_{L 3} & \ldots & A_{L L}
\end{array}\right)\left(\begin{array}{l}
T_{2} \\
T_{3} \\
\ldots \\
T_{L}
\end{array}\right)=\left(\begin{array}{l}
R_{2 \infty} \\
R_{3 \infty} \\
\ldots \\
R_{L \infty}
\end{array}\right)-\left(\begin{array}{l}
A_{21} T_{0} \\
A_{31} T_{0} \\
\ldots \\
A_{L 1} T_{0}
\end{array}\right)
$$

Example of non-uniform and uniform mesh distributions and element lengths are depicted in Figures 4 and 5 respectively.

\subsection{Example Problem 2: 1-D Convection Tip Thin Rectangular Fin}

When the base of the fin is held at a constant temperature, $T_{0}$ and the tip of the fin is a convection surface, then the boundary conditions are $T=T_{0} \quad$ at $x=0$

$$
q=h\left(T_{L}-T_{\infty}\right) \quad \text { at } \mathrm{x}=\mathrm{L}
$$

And the final global matrix shown in Equation (16) becomes

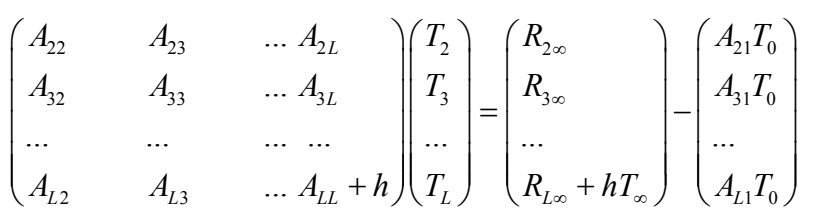

\section{Simulation Set-up and Assumptions}

Table 1 shows the considered parameters and their corresponding values used to obtain simulation results using FORTRAN 90 software. We used these values to obtain the temperature distribution for EFEM, CFEM, EDQM and exact methods.

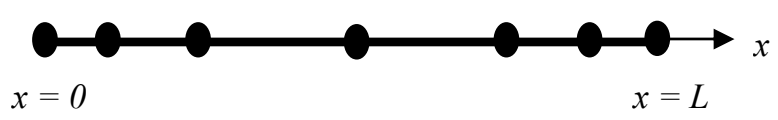

Figure 4: Example 1-D efficient mesh distribution and element lengths.

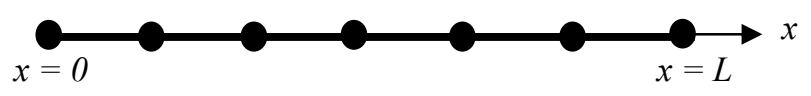

Figure 5. Example 1-D conventional mesh distribution and element lengths.
We considered, $M^{2}=\frac{h P}{k A}=1$ and the associated assumptions (in Table 1) to compare the obtained FEM results with DQM [13] and exact solution [18]. Here to mention that, to obtain 1-D DQM solutions, element material properties, fin-width and fin-thickness are not required (which is the shortcoming of the method). The errors in FEM and DQM solutions are computed compared to exact solution [18].

\section{Results and Discussions}

\subsection{Results and Discussions of 1-D Insulated Tip Thin Rectangular Fin}

The results of the present problem, shown in Figure 6, contain the maximum absolute percentage errors in the FEM and DQM solutions obtained with uniformly (conventional) and non-uniformly (efficient) distributed nodal (mesh) points. It is essential to know, how many mesh points (elements) are required to obtain a convergent FEM solution in the solution domain.

Hence, the comparison of convergence of fin-temperature in terms of maximum \% error versus number of nodal (mesh) points for CFEM, EFEM and EDQM solutions is shown in Figure 6. Initially, all the solutions in terms of maximum \% errors show a monotonic convergence with the increasing number of mesh points (shown $Z=11$ to 104 ). It is apparent that EFEM results show bit less accuracy for $Z \leq 30$ and similar accuracy for $Z \geq 30$ compared to EDQM, but yields result with higher accuracy, of one order of magnitude or more with increasing $Z$ compared to CFEM. EDQM converge up to $Z=100$ and then saturated, whereas the EFEM solutions converge smoothly for all $\mathrm{N}$ within the solution domain, showing best converging result at $Z=100$ and 101. On the other hand, uniform FEM (CFEM) results converge slowly throughout the solution domain and then diverge without showing the best results like EFEM. It happens due to the mesh point distribution strategy of equally spaced and unequally spaced nodal points in the computational domain and the inherited complexity to compute the stiffness matrix for equally spaced nodal points. Hence, the efficiency of EFEM results is apparent.

Figure 7 shows the convergent numerical and exact solutions (fin temperature) and the corresponding percentage errors for $N=100$ elements (FEM case) which is equivalent to $Z=101$ mesh points (both FEM and DQM cases). These results are obtained at an interval of $\Delta x=0.1$ along the fin length, $0 \leq x \leq 1$, using cubic 
Table 1. Input parameters and assumptions for 1-d rectangular fin.

\begin{tabular}{|c|c|c|}
\hline Input Parameters & $\begin{array}{l}\text { Assumed value for Insulated-Tip } \\
\text { Fin }\end{array}$ & $\begin{array}{l}\text { Assumed value for Convec- } \\
\text { tion-Tip Fin }\end{array}$ \\
\hline \multicolumn{3}{|l|}{ Boundary and other values: } \\
\hline Initial temperature $\left(T_{0}\right)$ & $1^{\circ} \mathrm{C}$ & $1^{\mathrm{O}} \mathrm{C}$ \\
\hline Ambient temperature $\left(T_{\infty}\right)$ & $0^{\circ} \mathrm{C}$ & $0^{\circ} \mathrm{C}$ \\
\hline Heat flux $(q)$ & 0 at $x=1$ & Variable \\
\hline$\%$ Error threshold $\left(e_{h}\right)$ & $0-0.1$ & $0-0.1$ \\
\hline $\begin{array}{l}\text { Element Type (NNODE): } \\
\text { Linear (for 1-D) }\end{array}$ & 2 & 2 \\
\hline Element material properties: & Variable to make $M=1$ & $7.03125 \mathrm{~W} /\left(\mathrm{m}^{0} \mathrm{C}\right)$ \\
\hline Thermal conductivity $\left(k_{e}=k\right)$ & $9 \mathrm{~W} / \mathrm{m}^{2}{ }^{0} \mathrm{C}$ & $9 \mathrm{~W} / \mathrm{m}^{20} \mathrm{C}$ \\
\hline Convective heat transfer coefficient $(h)$ & $0 \mathrm{~W} / \mathrm{m}^{3}{ }^{0} \mathrm{C}$ & $0 \mathrm{~W} / \mathrm{m}^{3}{ }^{0} \mathrm{C}$ \\
\hline \multicolumn{3}{|l|}{ Heat source $(Q)$} \\
\hline \multicolumn{3}{|l|}{ Element (Fin) dimension: } \\
\hline & Variable to make $M=1$ & Variable to make $M=1$ \\
\hline & $0.001 \mathrm{~m}$ & Variable to make $M=1$ \\
\hline thickness $(t)$ & $11-104$ & $11-104$ \\
\hline Number of elements $(N)$ & & \\
\hline
\end{tabular}

spline interpolation. It is seen that all the solutions are very close to exact solutions throughout the length of the fin with temperature variations $T_{0}=1^{0} C$ at $x=0 \mathrm{~m}$ to $T_{L}=0.648^{\circ} \mathrm{C}$ at $x=1.0 \mathrm{~m}$.

Figure 8 shows the percentage errors at the base of the fin $(x=0)$ are 0 for all solutions due to initial temperature $T_{0}=1^{0} \mathrm{C}$ (Figure 7). The percentages errors remain the same with EFEM except little bit increase (with maximum error $2.44 \times 10^{-6}$ ) at the middle of the fin due to nodal point distribution with maximum spacing there. Whereas, with CFEM, it increases gradually along the length of the fin with the maximum percentage error $1.9 \times 10^{-4}$ at the fin-tip $(x=1)$. In other case, the oscillations (instability) of DQM results appear clearly compared to FEM results. The average percentage error in CFEM, EDQM [13] and EFEM are $1.2 \times 10^{-4}$, $2.24 \times 10^{-6}$ and $1.12 \times 10^{-6}$ respectively, which shows approximately $99 \%$ and $49 \%$ improvements in EFEM results demonstrating its superiority over CFEM and EDQM.

\subsection{Results and Discussion of 1-D Convection Tip Thin Rectangular Fin}

Here the results exhibit the same nature like insulated-tip fin but yield results with higher accuracy, of two order of magnitude or more with increasing $Z$ due to different material properties and fin-thickness (as the FEM solution and its accuracy depend on fin dimension, materials used and associated boundary conditions).

In Figure 9, the comparison of convergence versus number of mesh points of exact, FEM and DQM solutions for convection-tip fin with uniform and non-uniform mesh distributions is shown. It is apparent that for all cases, the solutions converge smoothly for all $Z$ within the solution domain. The comparison shows similar results as in Figure 6 except EFEM yields result with higher accuracy, of one order of magnitude or more with increasing $Z$ (for $Z>20$ ) compared to that with CFEM. Here, EFEM results converge from $Z=80$ showing best result at $Z=90$ to 101, EDQM [13] shows similar results with some oscillations, whereas CFEM does not exhibit any best convergence. 


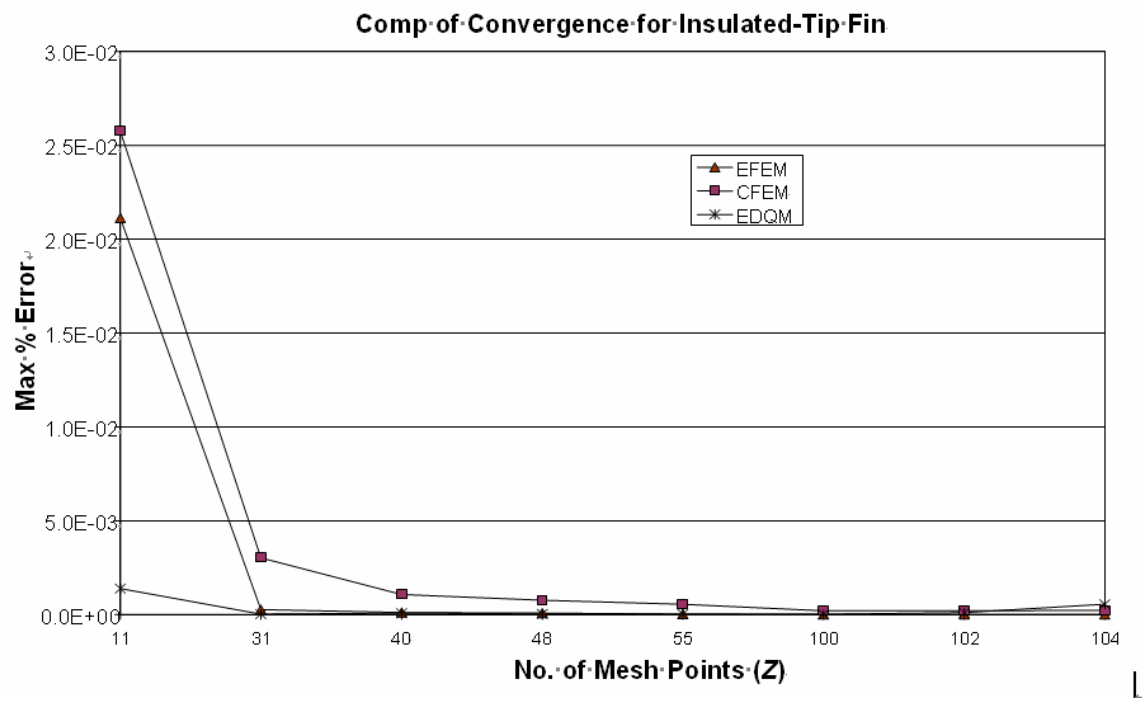

Figure 6. Comparison of convergence of insulated-tip fin-temperature in terms of maximum \% error for CFEM, EFEM and EDQM solutions ( $Z=11$ to 104 ).

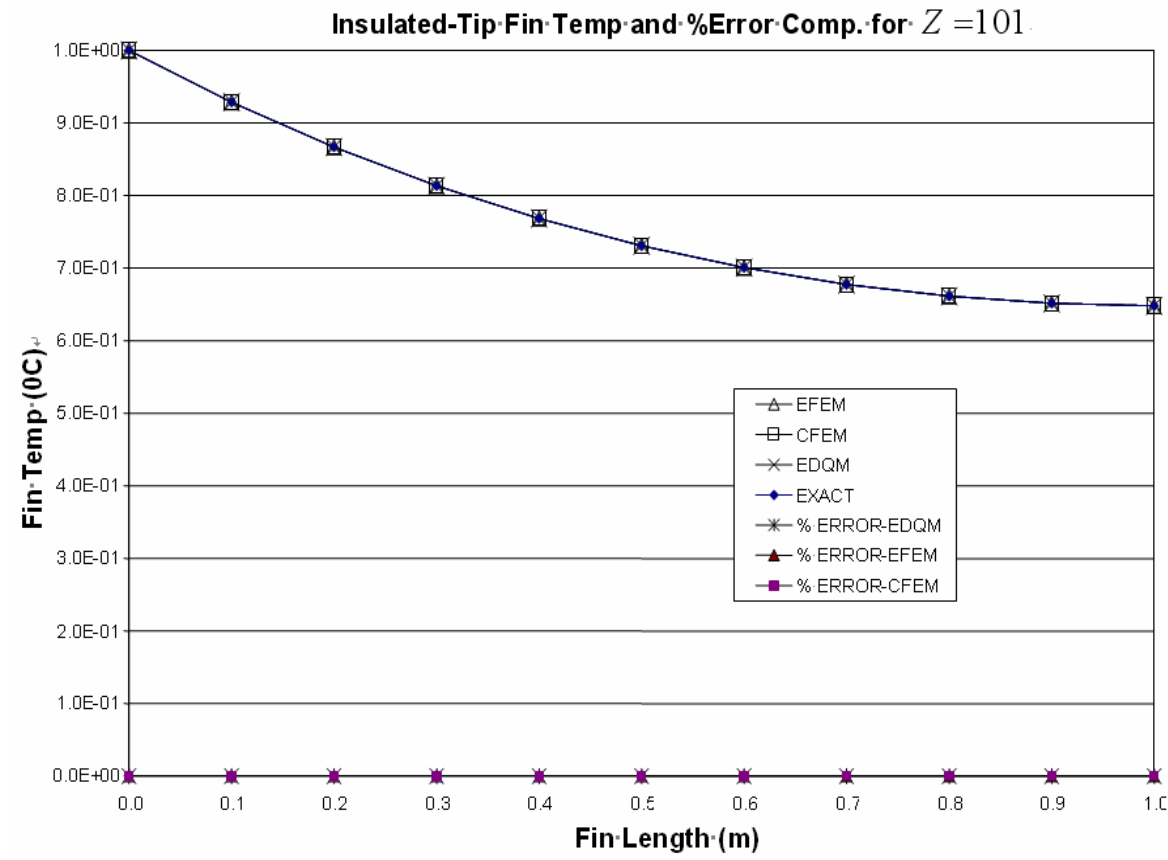

Figure 7. Insulated-tip fin-temperature distribution for exact, EFEM, CFEM and EDQM along with its respective \% errors $(Z=101)$.

Figure 10 depict the comparison of CFEM, EFEM, EDQM [13] numerical and exact convection-tip fin temperatures and the corresponding percentage errors for conventional (uniform) and efficient (non-uniform) mesh point distribution respectively for 100 elements (i.e., $Z=101)$. Same as Insulated-tip fin, the results are obtained at an interval of $\Delta x=0.1$ along the fin length, $0 \leq x \leq 1$, using cubic spline interpolation. Figure 10 shows that, all numerical solutions are very close to ex- act solutions throughout the length of the fin with temperature variations $T_{0}=1^{0} \mathrm{C}$ at base of the fin to $T_{L}=0.328^{0} \mathrm{C}$ at the tip of the fin. Here the reduction of fin temperature is $0.32^{\circ} \mathrm{C}$ more compared to insulated-tip fin (Figure 7) as expected.

FEM versus DQM maximum \% error comparison for convection-tip fin-temperature are shown in Figure 11. The comparison of CFEM, EFEM and EDQM [13] per- 


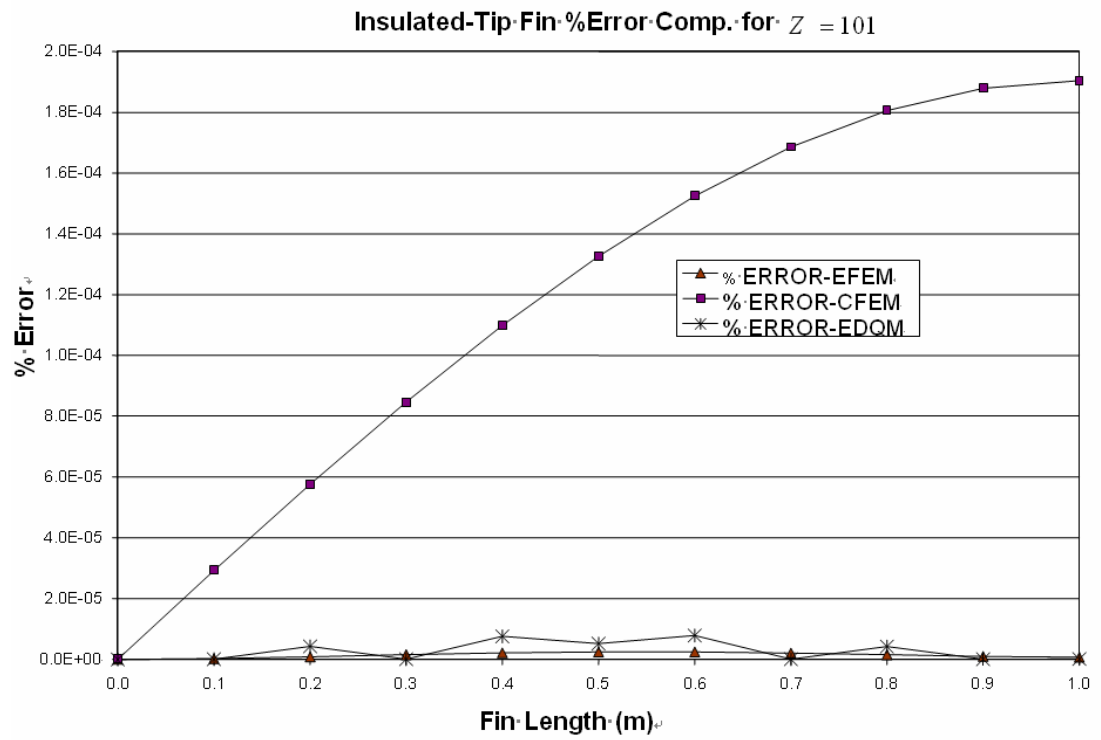

Figure 8. Percentage error comparison of EFEM, EDQM and CFEM for Z $=101$ along the fin-length.

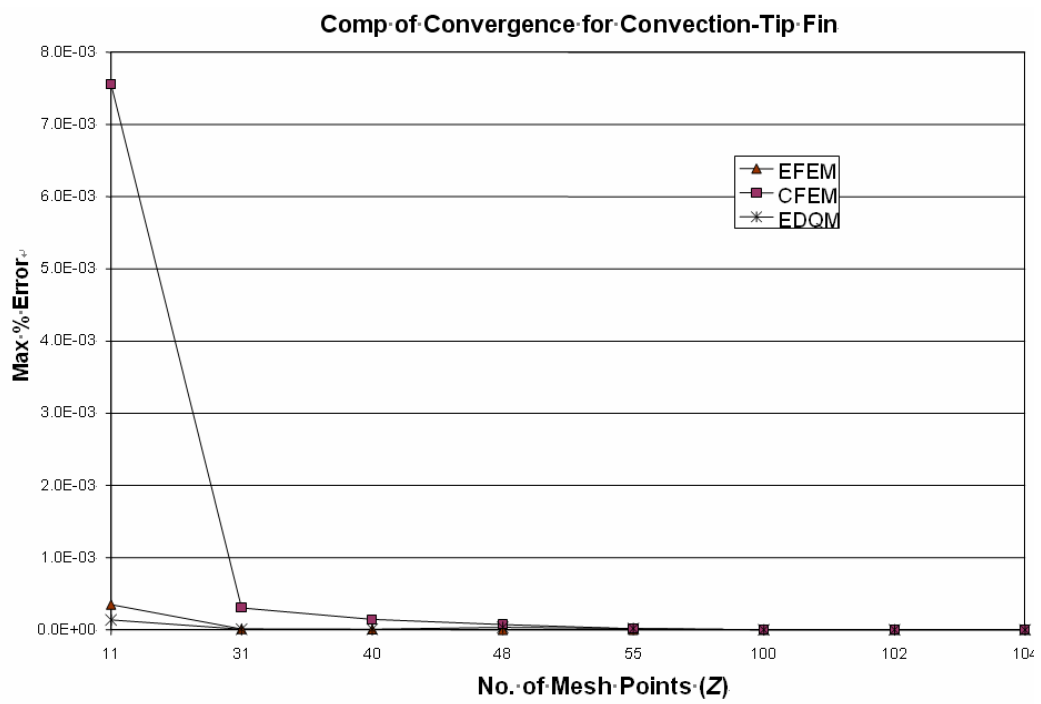

Figure 9. Comparison of convergence of convection-tip fin-temperature in terms of maximum \% error for CFEM, EFEM and EDQM solutions ( $Z=11$ to 104$)$.

centage errors for convection-tip fin is shown in Figure 11. There is no error at the base of the fin and it almost remain the same with EFEM and EDQM except negligible increase at the middle of the fin, whereas, with CFEM, it increases gradually along the length of the fin with the maximum percentage error $3.31 \times 10^{-6}$ at the tip $(x=1)$. In this case the EDQM converges with oscillations throughout the solution domain. The average \% error in CFEM, EDQM [13] and EFEM are $1.69 \times 10^{-6}$, $3.08 \times 10^{-9}$ and $2.24 \times 10^{-11}$ respectively. This shows nearly $100 \%$ and $99 \%$ improvements in EFEM results compared to CFEM and EDQM respectively demonstrat- ing its superiority.

\section{Conclusions}

Here, the solutions of the temperature distribution in insulated-tip and convection-tip 1-D rectangular fin are computed numerically using FEM and the results are found to agree very well with the exact solution and show the efficiency of the method. Investigating the various mesh points distribution for equal and unequal spacing, it is found that, for FEM solution, unequally spaced mesh points distribution give better and more 


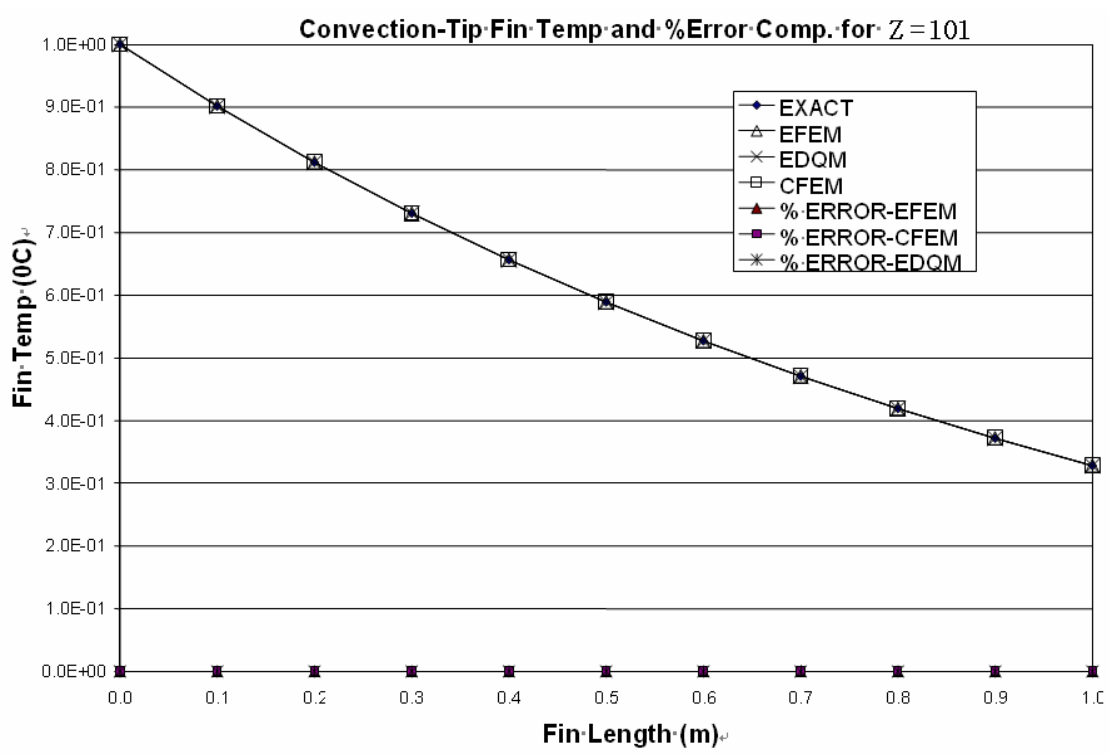

Figure 10. Convection-tip fin-temperature distribution for exact, EFEM, CFEM and EDQM along with its respective \% errors $(Z=101)$.

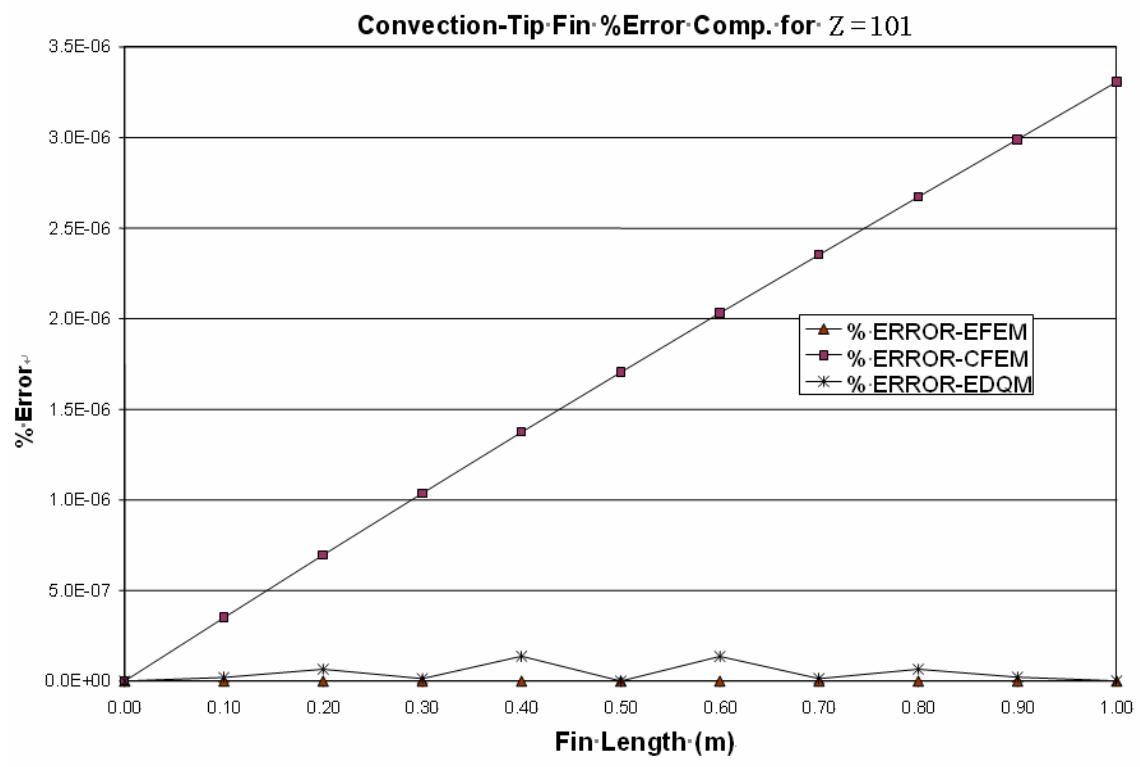

Figure 11. Convection-tip fin error comparison of EFEM, EDQM and CFEM for $Z=101$ along the fin-length.

accurate results than equally spaced and the solution converges smoothly as the number of nodal points (or elements) is increased. In general, this study has improved the stability and accuracy of EFEM results for practical consideration and implementation.

Finally, the results of EFEM shows remarkable enhancement compared to CFEM and agree very well with EDQM with very small errors or difference showing its potentiality. Hence EFEM is suitable to test the temperature distribution scenario in any thin metal fin prior to its design and practical implementation.

\section{References}

[1] G. Strang and G. J. Fix, "An analysis of the finite element method," Prentice-Hall, Inc., 1997.

[2] R. Tirupathi and P. E. Chandrupatla, Introduction to finite elements in engineering, Prentice-Hall International, 1997.

[3] "Mathematics of finite element method," 2004, Available at: http://math.nist.gov/mcsd/savg/tutorial/ansys/FEM/ (Accessed on October 19, 2006).

[4] B. Li, "Numerical method for a parabolic stochastic partial differential equation," Chalmers Finite Element Center, 2004. 
[5] F. Fairag, "Numerical computations of viscous, incompressible flow problems using a two-level finite element method," arXiv: Math.NA/0109109, Vol. 1, No. 17, September 2001.

[6] S. Park, "Development and applications of finite elements in time domain," PhD Thesis, Faculty of the Virginia Polytechnic Institute and State University, Virginia, December, 1996, Available in the Library, Virginia Polytechnic Institute and State University.

[7] R. Bellman and J. Casti, "Differential quadrature and long-term integration," J Math and Appl., Vol. 34, pp. 235-238, 1971.

[8] R. Bellman and J. Casti, "Differential quadrature: A technique for the rapid solution of nonlinear partial differential equations," J. Comput Phys, Vol. 10, pp. 40-52, 1972.

[9] C. W. Bert, S. K. Jang, and A. G. Striz, "Nonlinear bending analysis of orthotropic rectangular plates by the method of differential quadrature," J. Comput Mech, Vol. 5, pp. 217-226, 1989.

[10] C. W. Bert and M. Malik, "Fast computing technique for the transient response of gas-lubricated journal bearings," Proceedings of the U.S. National Congress of Applied Mechanics, Seattle, WA, pp. 298, June 26-July 1, 1994.

[11] C. Shu, W. Chen, H. Xue, and H. Du, "Numerical analysis of grid distribution effect on the accuracy of differential quadrature analysis of beams and plates by error estimation of derivative approximation," Int. Journal of Numer, Methods Engineering, Vol. 51, No. 2, pp. $159-179,2001$.
[12] M. M. Fakir, M. K. Mawlood, W. Asrar, S. Basri, and A. A. Omar, "Triangular fin temperature distribution by the method of differential quadrature," Journal Mekanikal, Malaysia, Vol. 15, pp. 20-31, 2003.

[13] M. M. Fakir, M. K. Mawlood, W. Asrar, S. Basri, and A. A. Omar, "Rectangular fin temperature distribution by the method of differential quadrature," The Journal of the Institute of Engineers, Malaysia (IEM), Vol. 63, No. 4, pp. 41-47, 2002.

[14] E. Hinton and D. R. J. Owen, "An introduction to finite element computations," Pineridge Press Limited, Swansea, U.K., 1985.

[15] S. Tiwari, G. Biswas, P. L. N. Prasad, and S. Basu, "Numerical prediction of flow and heat transfer in a rectangular channel with a built-in circular tube," ASME Journal of Heat Transfer, Vol. 125, No. 3, pp. 413-421, 2003.

[16] B. L. Wang, and Z. H. Tian, "Application of finite element-finite difference method to the determination of transient temperature field in functionally graded materials," Journal of Finite Elements in Analysis and Design, Vol. 41, pp. 335-349, 2005.

[17] S. H. Lo and W. X. Wang, "Finite element mesh generation over intersecting curved surfaces by tracing of neighbours," Journal of Finite Elements in Analysis and Design, Vol. 41, pp. 351-370, 2005.

[18] M. N. Ozisik, "Heat transfer: A basic approach," McGraw-Hill, 1985. 